\title{
Aus den Grenzgebieten
}

A. Marina: Das Ganglion eiliare ist das peripherische Zentrum Eür die

Lichtreaktion. Vortrag auf dem internationalen medizinischen Kongress in Budapest (September 1909).

Vortragender ist zu dem obigen Schluss sowohl auf Grund von Ex-perimenten wie auch von pathologisch-anatomischen Studien gekommen. Er will damit aber nicht sagen, dass das Ganglion eiliare das einzigc Zentrum für die Lichtreaktion sei.

Er halt es für möglich, dass die Konvergenzreaktion ,,primo loco” durch Dehnung der kurzen Ciliarnerven zustande komme, wahrschein-licher erscheint ihm jedoch die Annahme, dass Konvergenz- und Akkommodationsreaktion eine bis jetzt noch nicht ganz geklärte komplexe Ursache haben. (Nach dem Bericht im Neurol. Zentralblatt. 1909. S. 1115.) P. Sainton und J. Rathery: Troubles pupillairas et inégalité pupillaire temporaire à bascule dans le syndrome de Basedow. L'Encéphale 1908.

No. 7. Nach einem Referat aus dem Neurol. Zentralbl. 1909. S. 198. Die Verfasser unterscheiden vier Kategorien von Fallen:

!) Zusammengest $\theta$ llt von Groenouw in ßräfe-Sämisch, Handbuch der Augenheilk. II. Aufl. Zeitschrift für Augenheilkunde. Bd. XXIV. Heft 1. 37

558

Buchanzeige.

Fälle mit Dilatation der Pupillen, sie sind sehr häufig.

Fälle mit Miosis (selten).

Fälle mit Pupillenungleichheit (sehr selten).

Fälle, in denen der Mydriasis später eine Miosis folgte. Hiervon war bisher nur ein Fall beschrieben. Die Verf. bringen eine zweite Be-obachtung. Zur Erklärung nehmen sie eine toxisch-infektiöse Wirkung auf die dilatatorischen Irisfasern an.

$i_{i}{ }^{1} / 8 s-H a m b u r g:$ Ein Fall von wahrer paradoxer Lichtreaktion. Neurol. Zentralbl. 1909. S. 445.

59 jähriger Schmied, hatte vor 25 Jahren Lues. Im August 1908 und Dezember 1908

Schwindelanfall. Jetzt: allgemeine Herabsetzung der Sensibilität, links leichte Herabsetzung der Sehschärfe und Farben-empfindung, sonst an den Reflexen etc. nichts Krankhaftes. WassermannimBlutepositiv. Diagnose: Luescerebri. ,,DiemiotischenPupillen antworteten auf Lichteinfall direkt - nicht konsensuell - mit einer langsamen Erweiterung um das Doppelte bis Dreifache ihrer Grösse ohne vorausgehendeVerengerung, so oft derVorgang wiederholt wurde." M. Faure und Desvaulx: Das Argyll Robertsonsche Zeiehen bei Tabes.

Neurol. Zentralbl. 1909. S. 175.

Unter 200 Tabikern hatten nur 17 keine Pupillenstarre. Einmal fand sich einseitige Pupillenstarre, einmal schwand die ursprünglich vorhandene Pupillenstarre, 2 mal bestand paradoxe Reaktion, in 4 Fallen war die Reaktion verlangsamt, in 7 Fallen war sie vollständig normal. 
H. Lachmund: Untersuchungen über die Konvergenzreaktion bei reflek-toriseher Pupillenstarre. Berliner klin. Wochenschr. 1908. No. 27.

26 jähriges Mädchen, das an einer funktionellen Psychose erkrankte und während derselben eine rechtsseitige reflektorische Pupillenstarre darbot. Die rechte Pupille war weiter als die linke und blieb auch so, wenn sie sich auf Konvergenz verengert hatte. Auch die Entrundung bestand während der Konvergenzverengerung fort.

Der Verfasser halt die Erscheinung für angeboren. Er prüfte weiter das Verhalten der Konvergenzreaktion bei 27 Paralytikern und fand dieselbe bei 16 Pupillenpaaren prompt und ausgiebig, bei zwölf weiteren Paaren fand sich amSchluss der Verengerung Anisokorie und Entrundung (ein Teil der Fälle zeigte diese Erscheinungen übrigens schon vorher.)

Zur Erklärung der Abweichungen in der Weite und Form der Pupillen nimmt Verf. nicht eine Störung der Konvergenzreaktion selbst an, sondern er glaubt, dass die letztere ungestört verläuft und die er-wähnten Veränderungen durch andere, vielleicht noch unbekannte, Einflüsse auf die Pupille bedingt seien.

Hiibner--Bonn.

Buchanzeige.

Noe Scalinci-Neapel. La cataratta discrasica. Tipografia Fratelli Ruggiano. 1909.

I. In einer umf angreichen Monographie behandelt Scalinci die Pathogenese der Cataracta senilis und erörtert in der Einleitung die verschiedenen Theorien welche in den letzten 50 Jahren zur Erklärung der Linsentrübung aufgestellt worden sind (Austrocknung der Linse und Schrumpfung des 